\title{
New Type of Paranormed Behaviour of Spaces
}

\author{
Abdul Hamid Ganie ${ }^{1,2, *}$, Mashael M. AlBaidani ${ }^{3}$ \\ ${ }^{1}$ Department of Applied Sciences and Humanities, SSM College of Engineering and Technology, Pattan, Jammu and Kashmir, In- \\ dia. \\ ${ }^{2}$ Basic Science Department, College of Science and Theoretical Studies, Saudi Electronic University, Abha 6421, Asir Region, Sau- \\ di Arabia. \\ ${ }^{3}$ Department of Mathematics, College of Science and Humanities Studies, Prince Sattam Bin Abdulaziz University, Al Kharj 11942, \\ Riyadh Region, Saudi Arabia.
}

How to cite this paper: Abdul Hamid Ganie, Mashael M. AlBaidani. (2021) New Type of Paranormed Behaviour of Spaces. Journal of Applied Mathematics and Computation, 5(4), 273-276.

DOI: $10.26855 /$ jamc.2021.12.005

Received: September 8, 2021

Accepted: October 3, 2021

Published: November 3, 2021

*Corresponding author: Abdul Hamid Ganie, Department of Applied Sciences and Humanities, SSM College of Engineering and Technology, Pattan, Jammu and Kashmir, India; Basic Science Department, College of Science and Theoretical Studies, Saudi Electronic University, Abha 6421, Asir Region, Saudi Arabia.

Email: ashamidg@rediffmail.com

\begin{abstract}
Sequence spaces play a good role in summability fields in analysis. It was Kizmaz in H. Kizmaz, who introduced the concept of difference sequences spaces on $\delta_{\infty}, c$ and $c_{0}$ where $\int_{\infty} c$ and $c_{0}$ represents space of all bounded sequences, space of convergent sequence and the sequences converging to zero. In certain cases, the most general linear operator between two sequence spaces is given by an infinite matrix. So the theory of matrix transformations has always been of great interest in the study of sequence spaces. The sequences which were studied by Kizmaz were later studied by many authors and introduced different spaces. The authors in A. H. Ganie, et al. have recently studied the spaces $b v_{c}(g, p)$ and $b v_{0}(g, p)$ and interesting properties were analyzed. In this regard, the aim of this paper is to introduce the space $b v_{\infty}(g, p)$. It will be shown to be complete linear paranormed and prove that it is linearly isomorphic to the space $\int_{\infty}(p)$.
\end{abstract}

\section{Keywords}

Sequence space of non-absolute type, paranormed sequence space, infinite matrix

\section{Introduction}

Denoting the set of all sequences (real or complex) by $\Omega$, any subspace of $\Omega$ is called the sequence space. So the sequence space is the set of scalar sequences which is closed under co-ordinate wise addition and scalar multiplication. Throughout the paper $\mathbb{N}, \mathbb{R}$ and $\mathbb{C}$ denotes the set of non-negative integers, the set of real numbers and the set of complex numbers, respectively.

For a real linear space $X$, let $h$ be a function from $X$ to the set $\mathbb{R}$. Then, the pair $(X, h)$ is called a paranormed space and $h$ is a paranorm for $X$, if the following axioms are satisfied for all elements $\mathrm{u}, \mathrm{v} \in X$ and for all scalars $\alpha$ :

(i) $h(\theta)=0$

(ii) $h(-v)=h(v)$

(iii) $h(v+u) \leq h(v)+h(u)$ and

(iv) scalar multiplication is continuous.

Let $\delta_{\infty}, c$ and $c_{0}$ respectively be Banach spaces of bounded, convergent and null sequences $x=\left\{x_{n}\right\}_{n=0}^{\infty}$ normed by $\|x\|=\sup _{n \geq 0}|x(n)|$; also, as in I. J. Maddox [1], by $c s$ we denote the sequence of all convergent series.

Also, for a bounded sequence of strictly positive real numbers, we define 


$$
\operatorname{Lo}_{\infty}(p)=\left\{v=\left(v_{j}\right): \sup _{j}\left|v_{j}\right|^{p_{j}}<\infty\right\}
$$

Let $X$ and $Y$ be two non-empty subsets of the space $\omega$ of real or complex sequences. Let $A=\left(a_{n k}\right)$ be an infinite matrix of real or complex numbers. We write $(A x)_{n}=A_{n}(x)=\sum_{k} a_{n k} x_{k}$. Then $A x=\left\{A_{n}(x)\right\}$ is called the $A$-transform of $x$, whenever $A_{n}(x)=\sum_{k} a_{n k} x_{k}$ converges for each $n \in \mathbb{N}$. As in [2-4], we write $\lim _{n} A x=\lim _{n} A_{n}(x)$. If $x \in X$ implies $A x \in Y$, we say that $A$ defines a (matrix) transformation from $X$ into $Y$ and we denote it by $A: X \rightarrow Y$. By $(X: Y)$, we mean the class of all matrices $A$ such that $A: X \rightarrow Y$.

The approach of constructing a new sequence space by means of matrix domain of a particular limitation method has been studied by several authors as can be found in [5-8] and many others. In H. Kizmaz [9], the author has introduced the following difference sequence spaces $\mathrm{V}(\triangle)$ viz.,

$$
V(\triangle)=\left\{v=\left(v_{j}\right) \in \Omega:\left(\triangle v_{j}\right) \in V\right\},
$$

where $V \in\left\{\ell_{\infty}, c, c 0\right\}$ and $\Delta v_{j}=v_{j}-v_{j+1} \forall i \in \mathrm{N}$; note naught will be taken for a term with negative subscript.

\section{Main Section}

In this section, we shall introduce the space $b v_{\infty}(g, p)$ and prove some of its topological properties.

Following Mursaleen et al. [7], Ganie et al. [10-17], Naik et al. [18], Sheikh et al. [19], we define the space $b v_{\infty}(g, p)$ for a sequence $g=\left(g_{k}\right)$ with $g_{k} \neq 0$ for all $k \in \mathbb{N}$.

Incase $g_{k}=\mathrm{pk}=\mathrm{e}=(1,1, \ldots)$, it gets reduced to $\mathcal{L}_{\infty}(\triangle)$ [13].

Using (1), we may redefine the space $b v_{\infty}(g, p)$ as follows:

$$
\begin{aligned}
b v_{\infty}(g, p)=\left[\delta_{\infty}(p)\right]_{A^{g}}, \text { where } A^{g}=\left(a_{n k}^{g}\right) \text { with } \\
a_{n k}^{g}=\left\{\begin{array}{cc}
(-1)^{n-k} g_{n} & \text { if } n-1 \leq k \leq n, \\
0 & \text { if } 0 \leq k<n-1, \text { or } k>n,
\end{array}\right.
\end{aligned}
$$

for all $\mathrm{n}, \mathrm{k} \in \mathbb{N}$.

Theorem 2.1: The space $b v_{\infty}(g, p)$ is complete linear metric spaces paranormed by $f$ given by

$$
\oiiint(v)=\sup _{k}\left|g_{k} \Delta v_{k}\right|^{\frac{p_{k}}{M}} \text {. }
$$

Proof: The linearity of $b v_{\infty}(g, p)$ with respect to coordinate wise addition and scalar multiplication follows from the following inequality which are satisfied for $\xi, \varrho \in b v_{\infty}(g, p)$,

$$
\sup _{k}\left|g_{k} \Delta\left(\xi_{k}+\varrho_{k}\right)\right|^{\frac{p_{k}}{M}} \leq \sup _{k}\left|g_{k} \Delta \xi_{k}\right|^{\frac{p_{k}}{M}}+\sup _{k}\left|g_{k} \Delta \mathrm{Q}_{k}\right|^{\frac{p_{k}}{M}} \text {. }
$$

Now for any $\alpha \in \mathbb{R}$, we have

$$
|\alpha|^{p_{k}} \leq \max \left(1,|\alpha|^{H}\right)
$$

It is clear that $\mathrm{f}(\theta)=0$ and $\mathrm{f}(\mathrm{v})=\mathrm{f}(-\mathrm{v})$ for all $\mathrm{v} \in b v_{\infty}(g, p)$. Again, the inequalities (1) and (2) yield the subadditivity of $f$ and

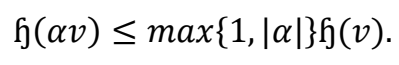

Thus, we have

$$
\mathfrak{f}\left(\alpha_{n} v_{n}-\alpha v\right)=\sup _{k}\left|g_{k} \Delta\left(v_{k}^{(n)}-\alpha v_{k}\right)\right|^{\frac{p_{k}}{M}} \leq \max \{1,|\alpha \mathrm{n}-\alpha|\} \mathfrak{f}(\mathrm{v})+|\alpha| \mathfrak{f}(\mathrm{vn}-\mathrm{v})
$$

which tends to zero as $n \rightarrow \infty$. That is to say that the scalar multiplication is continuous. Hence, $f$ is a paranorm on the space $b v_{\infty}(g, p)$.

It remains to prove the completeness of the space $b v_{\infty}(g, p)$. Let $\left\{v_{i}\right\}$ be any Cauchy sequence in the space $b v_{\infty}(g, p)$, where

$$
v^{i}=\left\{v_{0}^{(i)}, v_{1}^{(i)}, v_{2}^{(i)}, \ldots\right\} .
$$

Then, for given $\epsilon>0$, there exists a positive integer $n_{0}(\epsilon)$ such that

$$
\mathrm{h}\left(\mathrm{v}^{i}-\mathrm{v}^{j}\right)<\frac{\epsilon}{2}
$$

for all $i, j \geq n_{0}(\epsilon)$. Using definition of $\mathfrak{f}$, we obtain for each $\mathrm{k} \in \mathbb{N}$ that 


$$
\left|\left(A^{g} v^{i}\right)_{k}-\left(A^{g} v^{j}\right)_{k}\right|^{\frac{p_{k}}{M}} \leq \sup _{k}\left|\left(A^{g} v^{i}\right)_{k}-\left(A^{g} v^{j}\right)_{k}\right|^{\frac{p_{k}}{M}}<\frac{\epsilon}{2}
$$

for each $i, j \geq n_{0}(\epsilon)$. Consequently, $\left\{\left(A^{g} v^{0}\right)_{k},\left(A^{g} v^{1}\right)_{k},\left(A^{g} v^{2}\right)_{k}, \ldots\right\}$ is a Cauchy sequence of real numbers for every $\mathrm{k} \in \mathbb{N}$. But $\mathbb{R}$ is complete, it converges, say $\left(A^{g} v^{i}\right)_{k} \rightarrow\left(A^{g} v\right)_{k}$ as $i \rightarrow \infty$. Using these infinitely many limits, we have from (5) with $j \rightarrow \infty$ that

$$
\left|\left(A^{g} v^{i}\right)_{k}-\left(A^{g} v^{j}\right)_{k}\right|^{\frac{p_{k}}{M}}<\frac{\epsilon}{2}
$$

for every fixed $k \in \mathbb{N}$. Since $v^{i}=\left\{v_{k}^{(i)}\right\} \in b v_{\infty}(g, p)$ for each $i \in \mathbb{N}$, there exists $k_{0} \in \mathbb{N}$ such that

$$
\left|\left(A^{g} v^{i}\right)_{k}\right|^{\frac{p_{k}}{M}}<\frac{\epsilon}{2}
$$

for every $k \geq k_{0}$ and for each fixed $i \in \mathbb{N}$. Therefore, taking a fixed $i \geq n_{0}$, we see that

$$
\left|\left(A^{g} v^{i}\right)_{k}\right|^{\frac{p_{k}}{M}} \leq\left|\left(A^{g} v\right)_{k}-\left(A^{g} v^{j}\right)_{k}\right|^{\frac{p_{k}}{M}}+\left|\left(A^{g} v^{j}\right)_{k}\right|^{\frac{p_{k}}{M}}<\epsilon
$$

for every $k \geq k_{0}(\epsilon)$. This shows that $\mathrm{v} \in b v_{\infty}(g, p)$. Since $\left\{v_{i}\right\}$ was an arbitrary Cauchy sequence, the space $b v_{\infty}(g, p)$ is complete and this completes the proof.

Note that one can easily check that the absolute property does not hold for the space $b v_{\infty}(g, p)$, since there exists atleast one sequence in $b v_{\infty}(g, p)$ such that $\mathrm{f}(\mathrm{v}) \neq \hat{\mathrm{f}}(|\mathrm{v}|)$ where $|v|=\left(\left|v_{k}\right|\right)$. This means that $b v_{\infty}(g, p)$ is the sequence space of non-absolute type.

Theorem 2.2: The sequence space $b v_{\infty}(g, p)$ of non-absolute type is linearly isomorphic to the space $\int_{\infty}(p)$.

Proof: To prove the theorem, we should show the existence of a linear.

bijection between the spaces $b v_{\infty}(g, p)$ and $\mathcal{L}_{\infty}(p)$. With the notaion of (2), define the transformation $\mathrm{T}$ from $b v_{\infty}(g, p)$ to $\mathcal{L}_{\infty}(p)$ by $v \rightarrow y=\mathrm{T} v$. The linearity of $\mathrm{T}$ is trivial. Further, it is obvious that $v=\theta$ whenever $\mathrm{T} v=\theta$ and hence $\mathrm{T}$ is injective.

Let $y \in L_{\infty}(p)$ and define the sequence $v=\left\{v_{k}\right\}$ by

$$
v_{k}=\sum_{j=0}^{k} \frac{y_{j}}{g_{k}}, \quad(k \in \mathbb{N}) \text {. }
$$

Thus,

$$
\mathfrak{h}(v)=\sup _{k}\left|g_{k} \Delta v_{k}\right|^{\frac{p_{k}}{M}}=\sup _{k}\left|y_{k}\right|^{\frac{p_{k}}{M}}<\infty
$$

which shows that $v \in b v_{\infty}(g, p)$. Moreover, we observe that

$$
\lim _{k}\left|g_{k} \Delta\left(\sum_{j=0}^{k} \frac{y_{j}}{g_{k}}\right)\right|^{\frac{p_{k}}{M}}=\lim _{k}\left|g_{k} \frac{y_{k}}{g_{k}}\right|^{\frac{p_{k}}{M}}=\lim _{k}\left|y_{k}\right|^{\frac{p_{k}}{M}}=0 .
$$

Consequently, $\mathrm{T}$ is surjective and is norm preserving. Hence, $\mathrm{T}$ is a linear bijection and hence the spaces $b v_{\infty}(g, p)$ and $\delta_{\infty}(p)$ are linearly isomorphic as desired.

\section{Acknowledgement}

We are thankful for the reviewer(s) for the suggestions we received to improve the presentation of the paper.

\section{References}

[1] I. J. Maddox. (1967). Spaces of strongly summable sequences. Quart. J. Math. Oxford, 18(2), 345-355.

[2] B. Altay and F. Basar. (2003). On the space of sequences of p-bounded variation and related matrix mappings. Ukarnian Math. J., 1(1), 136-147.

[3] D. Fathima and A. H. Ganie. (2021). On some new scenario of Delta-spaces. Journal of Nonlinear Sciences and Applications, 14 (2021), 163-167. 
[4] A. H. Ganie and N. A. Sheikh. (2013). On some new sequence space of non-absolute type and matrix transformations. J. Egypt. Math. Soc., (2013), 34-40.

[5] K. G. Gross Erdmann. (1993). Matrix transformations between the sequence spaces of Maddox. J. Math. Anal. Appl., 180(1993), 223-238.

[6] I. J. Maddox. (1968). Paranormed sequence spaces generated by infinite matrices. Proc. Camb. Phil. Soc., 64(1968), 335-340.

[7] M. Mursaleen, A. H. Ganie, and N. A. Sheikh. (2014). New type of difference sequenence spaces and matrix transformation. Filomat, 28(7), 1381-1392.

[8] N. A. Sheikh and A. H. Ganie. (2012). A new paranormed sequence space and some matrix transformations. Acta Math. Acad. Paeda. Nyregy., 28(2012), 47-58.

[9] H. Kizmaz. (1981). On certain sequence, Canad. Math. Bull., 24(2), 169-176.

[10] A. H. Ganie. (2021). Some new approach of spaces of non-integral order. J. Nonlinear Sci, Appl., 14(2), 89-96.

[11] A. H. Ganie and D. Fathima. (2020). Almost convergence property of generalized Riesz spaces. Journal of Applied Mathematics and Computation, 4(4), 249-253.

[12] A. H. Ganie and N. A. Sheikh. (2015). Infinite matrices and almost convergence. Filomat, 29(6), 1183-1188.

[13] A. H. Ganie, N. A. Sheikh, and T. Jalal. (2015). Matrix transformations of some sequence spaces over non-archmedian fields. J. Appl. Computat. Math., (4), 1-3.

[14] A. H. Ganie, N. A. Sheikh, and T. Jalal. (2015). On some new spaces of invariant means with respect to modulus function, The inter. Jou. Modern Math. Sciences, USA, 13(3), 210-216.

[15] A. H. Ganie, N. A. Sheikh, and T. Jalal. (2014). New type of sequence spaces and matrix transformations. The inter. Jou. Modern Math. Sciences, USA, 10(3), 125-129.

[16] A. H. Ganie, Mobin Ahmad, N. A. Sheikh, T. Jalal, and S. A. Gupkari. (2016). Some new type of difference sequence space of non-absolute type, Int. J. Modern Math. Sci., 14(1), 116-122.

[17] A. H. Ganie. New spaces over modulus function. Boletim da Sociedade Paranaense de Matemática (in press), 1-6.

[18] P. A. Naik and T. A. Tarry. Matrix Representation of an All-inclusive Fibonacci Sequence. Asian J. Math. Stat., $11(1), 18-26$.

[19] N. A. Sheikh and A. H. Ganie. (2013). New paranormed sequence space and some matrix transformations. Int. J. Mod. Math. Sci., 8(2), 185-194. 